\title{
The Therapeutic Effect of Human Embryonic Stem Cell-Derived Multipotent Mesenchymal Stem Cells on Chemical-Induced Cystitis in Rats
}

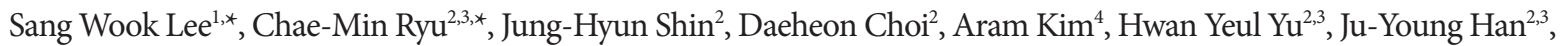 \\ Hye-Yeon Lee ${ }^{3,5}$, Jisun Lim $^{3,5}$, Yong Hwan Kim ${ }^{3,5}$, Jinbeom Heo ${ }^{3,5}$, Seungun Lee ${ }^{3,5}$, Hyein Ju ${ }^{3,5}$, Sujin Kim ${ }^{3,5}$, Ki-Sung Hong \\ Ji-Yeon $\mathrm{Han}^{7}$, Miho Song ${ }^{2}$, Hyung-Min Chung, ${ }^{6,8}$, Jun Ki Kim', Dong-Myung Shin ${ }^{3,5}$, Myung-Soo Choo ${ }^{2}$ \\ ${ }^{1}$ Department of Urology, Kangwon National University School of Medicine, Chunchon, Korea \\ ${ }^{2}$ Department of Urology, Asan Medical Center, University of Ulsan College of Medicine, Seoul, Korea \\ ${ }^{3}$ Department of Biochemical Science, Asan Medical Center, University of Ulsan College of Medicine, Seoul, Korea \\ ${ }^{4}$ Department of Urology, Konkuk University Medical Center, Konkuk University School of Medicine, Seoul, Korea \\ ${ }^{5}$ Department of Physiology, Asan Medical Center, University of Ulsan College of Medicine, Seoul, Korea \\ ${ }^{6}$ Department of Stem Cell Biology, Konkuk University School of Medicine, Seoul, Korea \\ ${ }^{7}$ Department of Urology, Pusan National University Yangsan Hospital, Pusan National University School of Medicine, Busan, Korea \\ ${ }^{8}$ Mirae Cell Bio Co. LTD., Seoul, Korea \\ ${ }^{9}$ Asan Institute for Life Sciences, Asan Medical Center, University of Ulsan College of Medicine, Seoul, Korea
}

Purpose: To evaluate the therapeutic effect of human embryonic stem cell (hESC)-derived multipotent mesenchymal stem cells (M-MSCs) on ketamine-induced cystitis (KC) in rats.

Methods: To induce KC, 10-week-old female rats were injected with 25-mg/kg ketamine hydrochloride twice weekly for 12 weeks. In the sham group, phosphate buffered saline (PBS) was injected instead of ketamine. One week after the final injection of ketamine, the indicated doses $\left(0.25,0.5\right.$, and $1 \times 10^{6}$ cells) of M-MSCs (KC + M-MSC group) or PBS vehicle (KC group) were directly injected into the bladder wall. One week after M-MSC injection, the therapeutic outcomes were evaluated via cystometry, histological analyses, and measurement of gene expression. Next, we compared the efficacy of M-MSCs at a low dose $\left(1 \times 10^{5}\right.$ cells $)$ to that of an identical dose of adult bone marrow (BM)-derived MSCs.

Results: Rats in the KC group exhibited increased voiding frequency and reduced bladder capacity compared to rats of the sham group. However, these parameters recovered after transplantation of M-MSCs at all doses tested. KC bladders exhibited markedly increased mast cell infiltration, apoptosis, and tissue fibrosis. Administration of M-MSCs significantly reversed these characteristic histological alterations. Gene expression analyses indicated that several genes associated with tissue fibrosis were markedly upregulated in KC bladders. However the expression of these genes was significantly suppressed by the administration of M-MSCs. Importantly, M-MSCs ameliorated bladder deterioration in KC rats after injection of a low dose $\left(1 \times 10^{5}\right)$ of cells, at which point BM-derived MSCs did not substantially improve bladder function.

Conclusions: This study demonstrates for the first time the therapeutic efficacy of hESC-derived M-MSCs on KC in rats. MMSCs restored bladder function more effectively than did BM-derived MSCs, protecting against abnormal changes including mast cell infiltration, apoptosis and fibrotic damage.

Keywords: Cystitis; Fibrosis; Ketamine; Multipotent stem cells; Pelvic pain

Corresponding author: Myung-Soo Choo (iD http://orcid.org/0000-0002-3293-4751 Department of Urology, Asan Medical Center, University of Ulsan College of Medicine, 88 Olympic-ro 43-gil, Songpa-gu, Seoul 05505, Korea

E-mail: mschoo@amc.seoul.kr / Tel: +82-2-3010-2086 / Fax: +82-2-3010-8493

Co-corresponding author: Dong-Myung Shin (D) https://orcid.org/0000-0002-0511-5750

Department of Biomedical Sciences, Physiology, Asan Medical Center, University of

Ulsan College of Medicine, 88 Olympic-ro 43-gil, Songpa-gu, Seoul 05505, Korea

E-mail: d0shin03@amc.seoul.kr / Tel: +82-2-3010-2086 / Fax: +82-2-3010-8493

*Sang Wook Lee and Chae-Min Ryu contributed equally to this study as co-first authors.

Submitted: January 11, 2018 / Accepted after revision: January 22, 2018

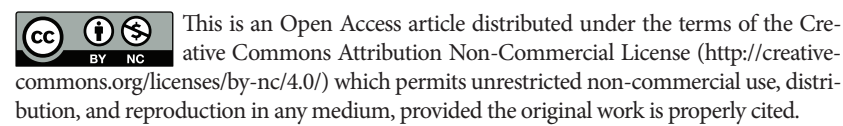
bution, and reproduction in any medium, provided the original work is properly cited. 
- Grant/Fund Support: This research was supported by grants from the Korean Health Technology R\&D Project, Ministry of Health \& Welfare, Republic of Korea (grant no. HI14C2321 and HI14C3365), by the National Research Foundation of Korea (NRF-2017M3A9B4061890), and by grants (2017-528 and 2017-098) from the Asan Institute for Life Sciences, Asan Medical Center, Seoul, Korea.

- Research Ethics: All animal experiments were performed in accordance with the guidelines and regulations of the institution and were approved by the Institutional Animal Care and Use Committee of the University of Ulsan College of Medicine (IACUC-2016-12-088).

- Conflict of Interest: No potential conflict of interest relevant to this article was reported.

\section{- HIGHLIGHTS}

- Administration of hESC-derived M-MSCs significantly restored bladder function and reversed characteristic histological alterations of KC bladders in rats.

- M-MSCs ameliorated bladder function of KC rats at a low dose with superior efficacy compared to adult BM-derived MSCs.

\section{INTRODUCTION}

In recent years, the abuse of ketamine has dramatically increased worldwide, particularly in terms of recreational use [1]. Long-term ketamine abuse can trigger severe lower urinary tract symptoms and bladder pain, complicated by a reduced bladder capacity (BC) and hematuria [2-4]. A recent study found that urinary tract symptoms were reported by $>25 \%$ of all regular ketamine users [2].

Although the pathophysiology and causative mechanism of the changes in bladder function remain unclear, the typical gross pathological bladder changes in ketamine-induced cystitis $(\mathrm{KC})$ are contraction and wall thickening [5]. Ulcerative cystitis with an easily bleeding mucosa is a common cystoscopic finding. Microscopically, the urothelium is denuded and infiltrated by inflammatory cells such as mast cells and eosinophils. These cystoscopic and histological findings, and also the lower urinary tract symptoms, resemble those of patients with interstitial cystitis (IC) [6], which is probably a severe form of bladder pain syndrome (BPS). The clinical and pathological similarities between $\mathrm{KC}$ and IC suggest that $\mathrm{KC}$ shares at least some pathogenic pathways with IC.

Therefore, an increased understanding of KC might also improve our understanding of IC pathogenesis. In addition, therapeutic targeting of pathways common to KC and IC pathogenesis might allow the development of novel treatment options for the obstinate painful bladder conditions.

The management of KC remains challenging. No single definitive treatment has yet been accepted because the pathophysiology of KC remains unclear [5]. Treatment should commence with cessation of ketamine use. Current therapeutic options for KC (also used to treat IC) include pain control medications, anticholinergics [6], urothelium-protecting agents [7], and intra- vesical injections of botulinum toxin [8]. Supratrigone cystectomy combined with enterocystoplasty is the only effective treatment for irreversible KC [3,9]. All of these options are of limited efficacy and/or exhibit other problems.

In recent years, stem cell therapies have opened up new pathways toward the therapy of several previously intractable disorders $[10,11]$. Stem cell-based therapy has been considered a novel therapeutic approach when seeking to resolve disorders of lower urinary tract including stress urinary incontinence, detrusor overactivity or underactivity, and bladder or urethral injury $[12,13]$. Injected stem cells stimulate the regeneration of damaged tissues and also secrete paracrine factors and cytokines promoting cellular survival and preventing apoptosis [14]. Recently, preclinical studies have revealed the beneficial effects of mesenchymal stem cells (MSCs) derived from human umbilical cord-blood (UCB) in rat models of IC [15] and KC [16]. As no curative treatments for BPS/IC or KC are yet available [17], stem cell-based therapy would be valuable for patients with BPS/IC or KC.

MSCs are multipotent progenitor cells. Clinically, they are safe and afford functional recovery from various human diseases $[10,18]$. They have been isolated from a wide range of adult tissues including bone marrow (BM), adipose tissue, dental pulp, and UCB [19]. However, their limited proliferative capacity during ex vivo expansion constitutes a major problem in terms of wider clinical applications. An alternative source of MSCs is required.

Human embryonic stem cells (hESCs) are an alternative cellular source of MSCs [20]. ESC lines established from the inner cell mass of the blastocyst can differentiate into all possible types of cells and can be expanded ex vivo in an immortalized manner [21]. Given this capacity for unlimited self-renewal, pluripotent hESCs are an attractive cellular resource for appli- 
cations in regenerative medicine [21,22]. A Korean research group recently described a simple and feasible method by which multipotent MSCs (M-MSCs) can be generated from hESCs [23,24]. These M-MSCs are available in virtually unlimited quantities and their differentiation can be controlled to optimize safety and potency prior to transplantation, overcoming the drawbacks of existing MSC therapy.

The purpose of this study was to evaluate the therapeutic effect of hESC-derived M-MSCs on KC in rats. We analyzed the cystometric parameters as well as the histologic and immunohistochemical findings. The expression levels of genes possibly associated with KC pathogenesis were also assessed.

\section{MATERIALS AND METHODS}

\section{Study Design}

The schematic diagram of the main study design is depicted in Fig. 1. Interventions involved a single administration of hESCderived M-MSCs at the indicated doses $\left(0.25,0.5\right.$, and $1 \times 10^{6}$ cells) in the experimental group or phosphate buffered saline (PBS) in the control group. The therapeutic outcomes were evaluated via awake cystometry, histological analyses, and measurement of gene expression. Next, we also compared the efficacy of M-MSCs at a low dose $\left(1 \times 10^{5}\right.$ cells $)$ to that of an identical dose of adult BM-derived MSCs with regard to cystometric parameters.

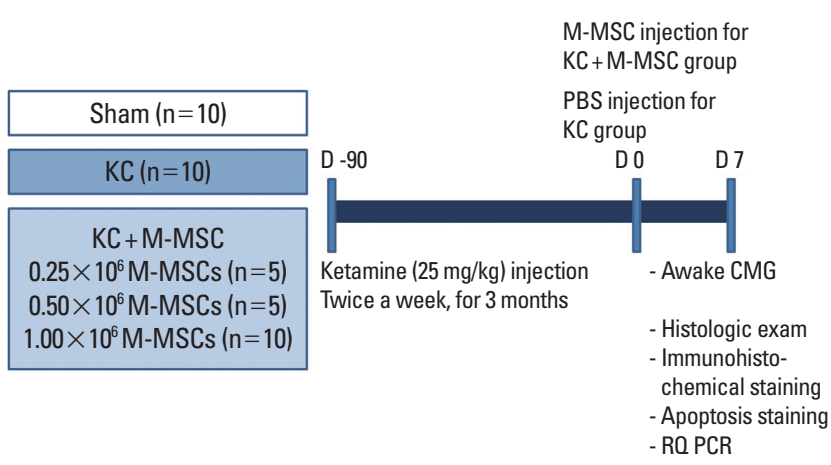

Fig. 1. Schematic diagram of the main study design. The control group (KC group) and the experimental group (KC+M-MSC group) were given ketamine twice weekly for 12 weeks. Interventions involved a single administration of human embryonic stem cell-derived multipotent mesenchymal stem cells (M-MSCs) at the indicated doses $\left(0.25,0.5\right.$, and $1 \times 10^{6}$ cells). One week after M-MSC injection, therapeutic outcomes were evaluated. KC, ketamine-induced cystitis; M-MSC, multipotent mesenchymal stem cell; PBS, phosphate buffered saline: CMG, cystometrography; RQ PCR, real-time quantitative polymerase chain reaction.

\section{Differentiation and Culture of hESC-derived M-MSCs}

Undifferentiated H9-hESCs were maintained and differentiated into M-MSCs as previously described [23,24]. M-MSCs were cultured in EGM2-MV medium (Lonza, San Diego, CA, USA) onto plates coated with rat tail collagen type I (Sigma-Aldrich, St. Louis, MO, USA) in a humidified atmosphere under $5 \% \mathrm{CO}_{2}$ at $37^{\circ} \mathrm{C}$. All M-MSCs were expanded for fewer than 10 passages to ensure that multipotency was preserved. Basic M-MSC features such as the surface protein profile, cell proliferation, multipotency (in vitro differentiation into osteogenic, chondrogenic, or adipogenic lineages), in vitro angiogenesis assays, and karyotype were evaluated as previously described $[23,24]$.

\section{Animal Models and Administration of M-MSCs}

All animal experiments were performed in accordance with the guidelines and regulations of the institution and were approved by the Institutional Animal Care and Use Committee of the University of Ulsan College of Medicine (IACUC-2016-12-088).

To induce KC, 10-week-old female Sprague-Dawley rats (OrientBio, Gapyong, Korea) were given ketamine hydrochloride (Huons, Seongnam, Korea; catalog No. EK1352-11) at 25$\mathrm{mg} / \mathrm{kg}$ alternately intravenously and intraperitoneally twice weekly for 12 weeks. In the sham group, PBS was injected instead of ketamine. One week after the final injection of ketamine, a lower abdominal incision was created in each rat and the indicated doses of M-MSCs (KC+M-MSC group) or PBS (KC group) were directly injected into the submucosal layer of the anterior wall or dome of the bladder using a $500-\mu \mathrm{m}$ syringe attached to a $26-\mathrm{G}$ needle. Therapeutic outcomes were evaluated 1 week later; we performed conscious cystometry and histological and gene expression analyses.

\section{Unanesthetized Unrestrained Cystometrography (Awake Cystometry)}

Cystometrograms were obtained from unanesthetized unrestrained rats in metabolic cages. Catheterizations allowing measurement of intravesical pressure (IVP) and intra-abdominal pressure (IAP) were performed 3 days prior to cystometrography, as described previously $[25,26]$. The bladder was accessed using an inflatable PE-50 catheter (Clay Adams, Parsippany, NJ, USA) connected to a pressure transducer (Research Grade Blood Pressure Transducer, Harvard Apparatus, Holliston, MA, USA) and a microinjection pump (PHD22/2000 pump; Harvard Apparatus). Micturition volumes (MVs) were continuously recorded using a fluid collector connected to a force displace- 
ment transducer (Research Grade Isometric Transducer, Harvard Apparatus) as sterile saline was infused into the bladder at a rate of $0.4 \mathrm{~mL} / \mathrm{min}$. The IVP, IAP, and MVs were continuously recorded using Acq Knowledge 3.8.1 software and an MP150 data acquisition system (Biopac Systems, Goleta, CA, USA) at a sampling rate of $50 \mathrm{~Hz}$.

\section{Histological and Immunohistochemical Analyses}

After 24 hours of fixation in 4\% paraformaldehyde, each bladder was embedded in paraffin and cut into 3 - $\mu$ m-thick slices that were affixed to slides and stained with hematoxylin and eosin. Mast cell infiltration and fibrosis were assessed via Toluidine Blue staining (Toluidine Blue-O, Daejung Chemicals \& Metals Co., Seoul, Korea) and Masson's Trichrome staining (Junsei Chemical, Tokyo, Japan), respectively. To assess apoptosis, bladder sections were stained with antibodies specific for terminal dUTP nick-end labeling (TUNEL) (Roche, Mannheim, Germany), followed by visualization using Alexa488-conjugated anti-mouse or rabbit antibodies (Molecular Probes, Grand Island, NY, USA). Quantitative digital image analyses were performed on seven randomly chosen representative areas of each slide. To quantify fibrosis and apoptosis, the areas staining with Masson's Trichrome and the TUNEL reagent were calculated using Image Pro 5.0 software (Media-Cybernetics, Rockville, MD, USA). Mast cell infiltration was quantified by counting cells that stained with Toluidine Blue.

\section{Reverse Transcriptase and Real-time Quantitative PCR}

Total RNA from the bladder tissues was isolated using the RNeasy-Mini Kit (QIAGEN, Valencia, CA, USA). Genomic DNA was extracted using a DNA-free Kit (Applied Biosystems, Foster City, CA, USA). mRNA (400 ng) was reverse-transcribed using a Taqman Reverse-Transcription-Reagents kit (Applied Biosystems) following the manufacturer's instructions. The expression levels of target genes were quantified using real-time quantitative PCR (RQ-PCR) employing the iQ5 Optical System (BIO-RAD, Hercules, CA, USA) with SYBR Green PCR Master Mix (BIO-RAD), as described previously [27-29]. The expression levels of genes involved in inflammation, apoptosis, and fibrosis were assessed. Sequences of the primers used in this study were described in Table 1.

\section{Statistical Analysis}

Data were reported as the mean \pm standard error of the mean (SEM) and were analyzed by GraphPad Prism 6.0 software
Table 1. Sequences of the primers in RQ-PCR

\begin{tabular}{rll}
\hline No. & Oligo name & \multicolumn{1}{c}{ Oligo sequence } \\
\hline 1 & rTgfb1_qRT_F & AGT TCA AGC AGA GTA CAC ACA GCA T \\
2 & rTgfb1_qRT_R & GAG AGC AAC ACG GGT TCA GGT A \\
3 & rTgfb2_qRT_F & CCC ATC TCA TTG CTC CAA GAA T \\
4 & rTgfb2_qRT_R & AAA AGG CCA GTA GTT CCA AAA GTG \\
5 & rTgfb3_qRT_F & AGA CCC CAC GTG CGA CAG A \\
6 & rTgfb3_qRT_R & AGG TTT GTT GCT TGT GTG TTT CC \\
7 & rSMAD2_qRT_F & GTG ATC CCG TGG ACT GTG CTA \\
8 & rSMAD2_qRT_R & CTC CAC AAG GTG CTT TAA CTG ATG \\
9 & rSMAD3_qRT_F & CCA TCC GCA TGA GCT TCG T \\
10 & rSMAD3_qRT_R & ACT GCA AGG GCC CAT TCA \\
11 & rSnai2_qRT_F & GCT TTT GCA GAC AGA TCA AAC CT \\
12 & rSnai2_qRT_R & GGA GCA GTT TTT GCA CTG GTA TT \\
13 & rTnfa_qRT_F & AGG CGC TCC CCA AAA AGA \\
14 & rTnfa_qRT_R & CCA CGA GCA GGA ATG AGA AGA \\
15 & rCxcl10_qRT_F & GGC CAT AGG AAA ACT TGA AAT CA \\
16 & rCxcl10_qRT_R & CAT TGT TCT TCT TCA TTG TGG CAA T \\
17 & rCard10_qRT_F & CGG AGG AAG CCA CAG ACA GT \\
18 & rCard10_qRT_R & TGC CGG CGG AGT ATG GA \\
19 & rIL4_qRT_F & GAG AAG CTG CAC CGT GAA TG \\
20 & rIL4_qRT_R & TCC CTC GTA GGA TGC TTT TTA GG \\
21 & rIL10_qRT_F & CCC TGG GAG AGA AGC TGA AGA \\
22 & rIL10_qRT_R & CCA CTG CCT TGC TTT TAT TCT CA \\
\hline &
\end{tabular}

RQ PCR, real-time quantitative polymerase chain reaction.

(GraphPad Software, La Jolla, CA, USA). Differences and significance were verified using 1-way analysis of variance followed by Bonferroni post hoc testing. P-values $<0.05$ were considered statistically significant.

\section{RESULTS}

\section{Evaluation of Bladder Function Using Cystometry}

Rats injected with ketamine over 3 months (the KC group) exhibited increased and irregular voiding frequencies and a decreased micturition interval (MI) compared to sham group rats (52.3 \pm 12.8 seconds vs. $149.5 \pm 26.5$ seconds, respectively, $\mathrm{P}<$ 0.001) (Fig. 2A, B). A transplantation of M-MSCs $(0.25,0.5$, and $1 \times 10^{6}$ cells; KC + M-MSC group) significantly increased the MI $(87.7 \pm 6.2,109.4 \pm 24.4$, and $151.8 \pm 35.1$ seconds, respectively, $\mathrm{P}<0.001)$. BC $(0.3 \pm 0.1 \mathrm{~mL}$ vs. $1.0 \pm 0.2 \mathrm{~mL}$, respectively, $\mathrm{P}<0.001)$ and $\mathrm{MV}(0.3 \pm 0.1 \mathrm{~mL}$ vs. $0.6 \pm 0.1 \mathrm{~mL}$, respec- 

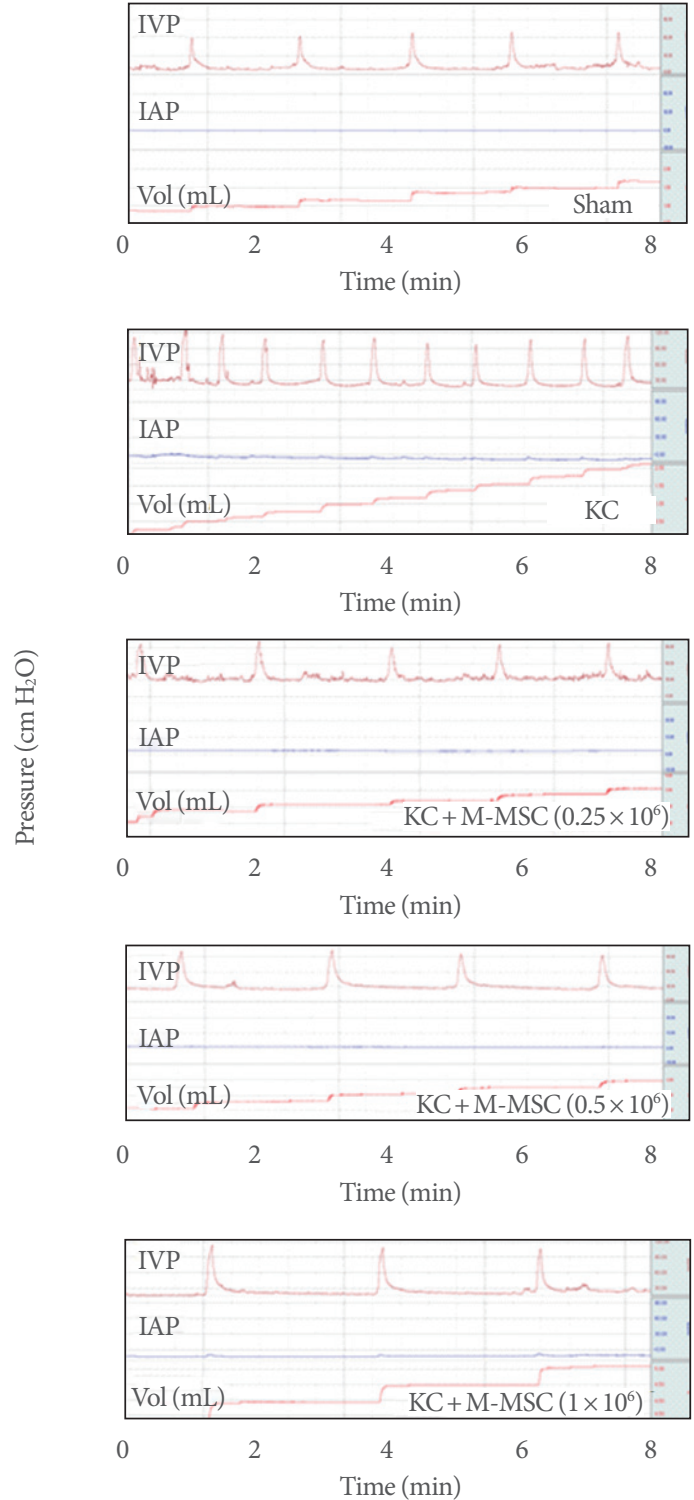
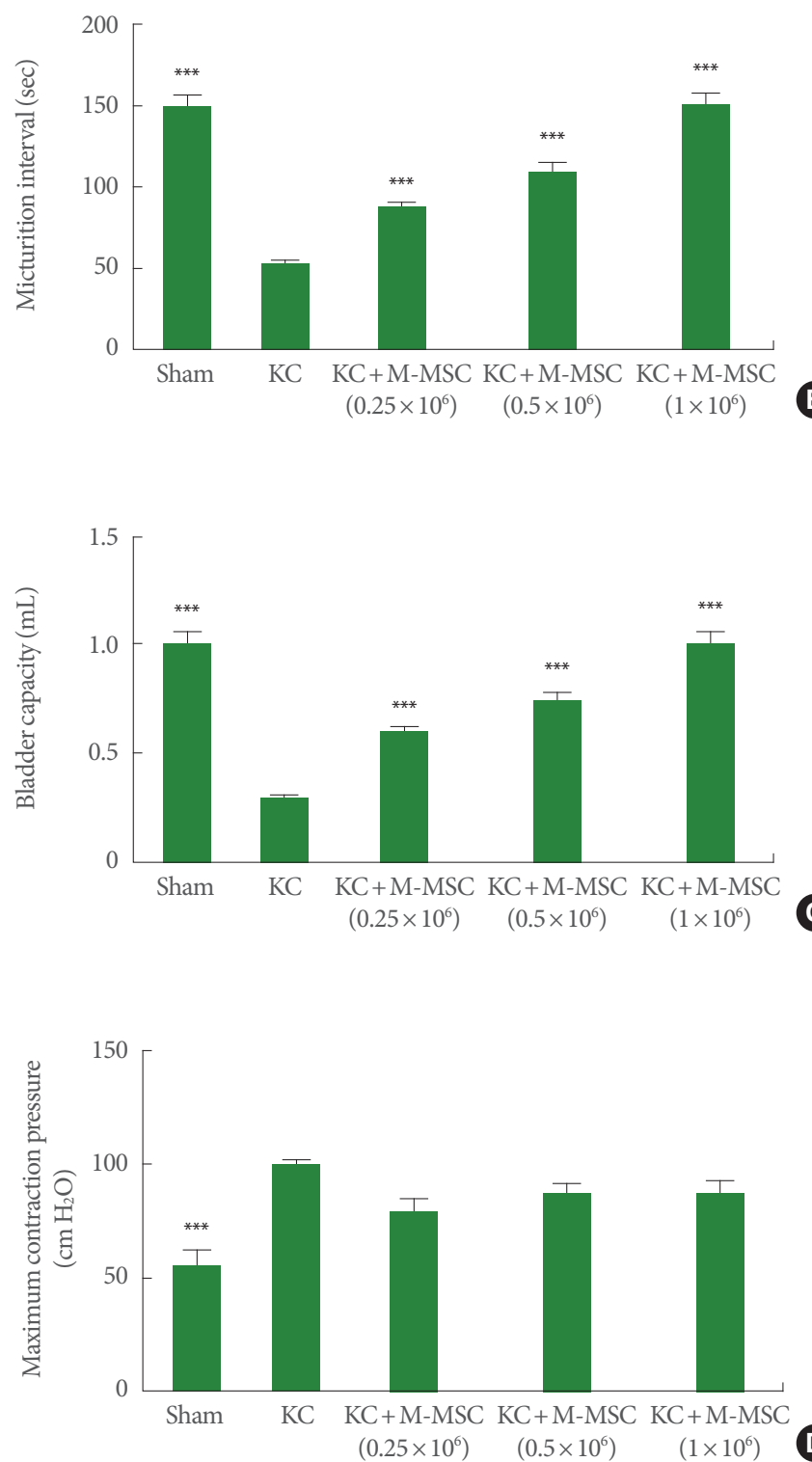

Fig. 2. Administration of M-MSCs improved the bladder function. (A) Representative cystometry results of the indicated groups. The micturition intervals (B), bladder capacity (C), and maximum contraction pressure (D) were quantified from the voiding pattern analysis. IVP, intravesical pressure; IAP, intra-abdominal pressure; KC, ketamine-induced cystitis; M-MSC, multipotent mesenchymal stem cell. ${ }^{* * *} \mathrm{P}<0.001$ compared with the KC group with Bonferroni posttest.

tively, $\mathrm{P}<0.001$ ) of rats in the KC group decreased significantly compared to those in the sham group. However, these parameters were reversed after transplantation of M-MSCs at all doses tested (Fig. 2C). In addition, basal pressure and maximum contraction pressure increased significantly in KC rats compared to sham rats. M-MSC injection did not significantly reverse this effect at any dose tested (Fig. 2D).

Compared to the same dose of adult BM-derived MSCs, a low dose $\left(1 \times 10^{5}\right)$ of M-MSCs exhibited superior in vivo therapeutic potency. M-MSCs ameliorated the bladder function of $\mathrm{KC}$ rats at a dose of $1 \times 10^{5}$ cells; at this dose, BM-derived MSCs did not substantially improve aberrant bladder function (Fig. 3).

Histological Analysis of the Beneficial Effects of M-MSC on KC As previously reported, KC bladders exhibited markedly increased infiltration of Toluidine Blue-staining mast cells (Fig. 4) 

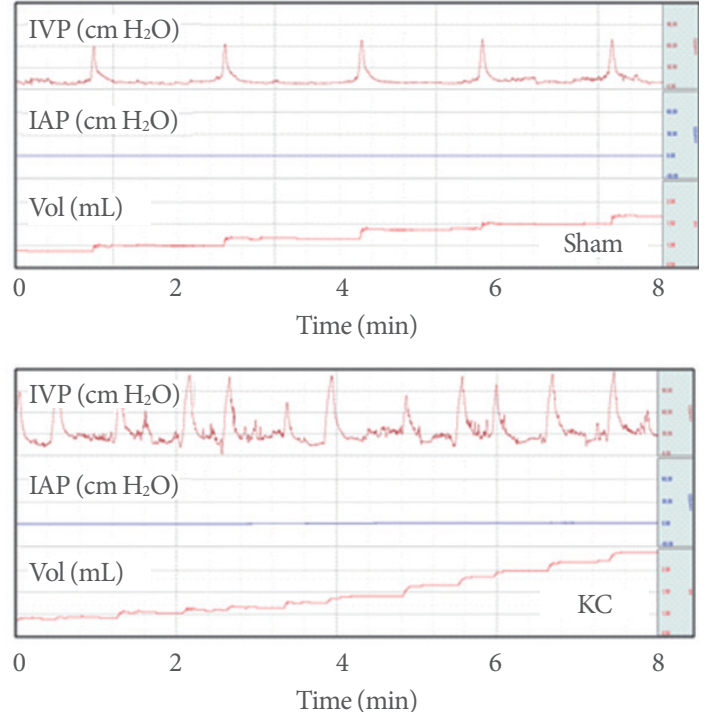
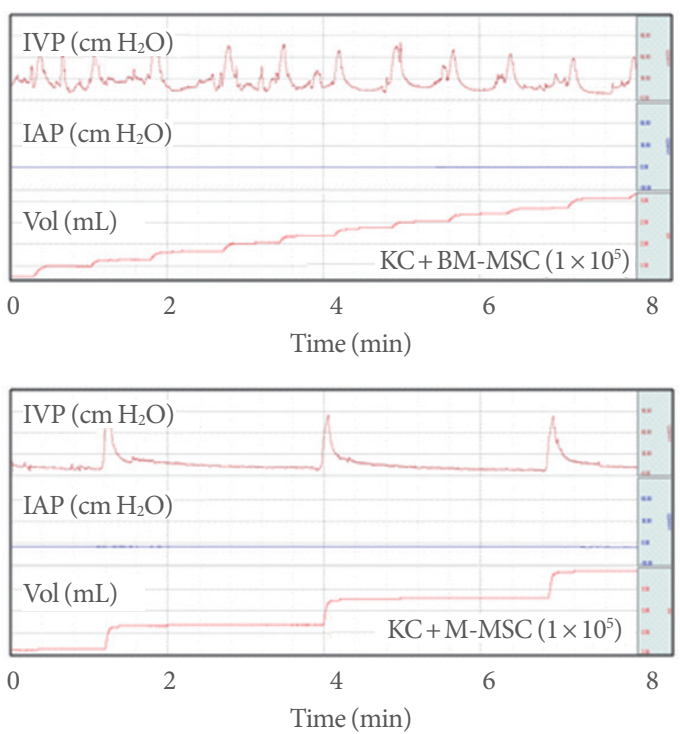

Fig. 3. Superior therapeutic efficacy of M-MSCs compared to BM-MSCs at a low dose $\left(1 \times 10^{5}\right.$ cells $)$. Representative cystometry results of the indicated groups are shown. IVP, intravesical pressure; IAP, intra-abdominal pressure; KC, ketamine-induced cystitis; MMSC, multipotent mesenchymal stem cell; BM-MSCs, bone marrow mesenchymal stromal cells.
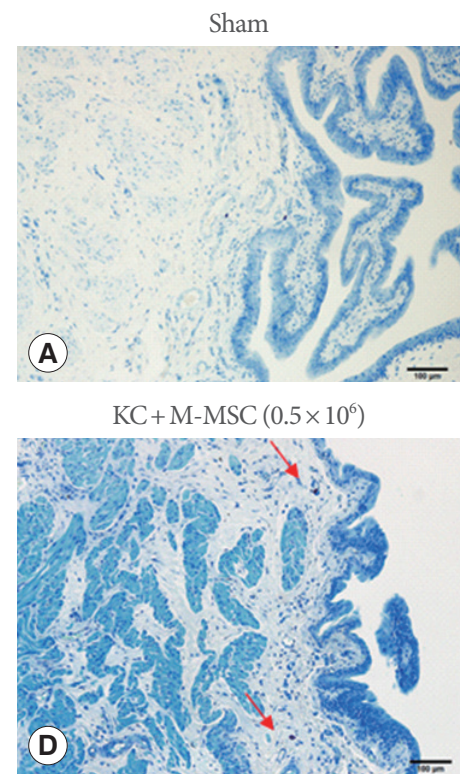
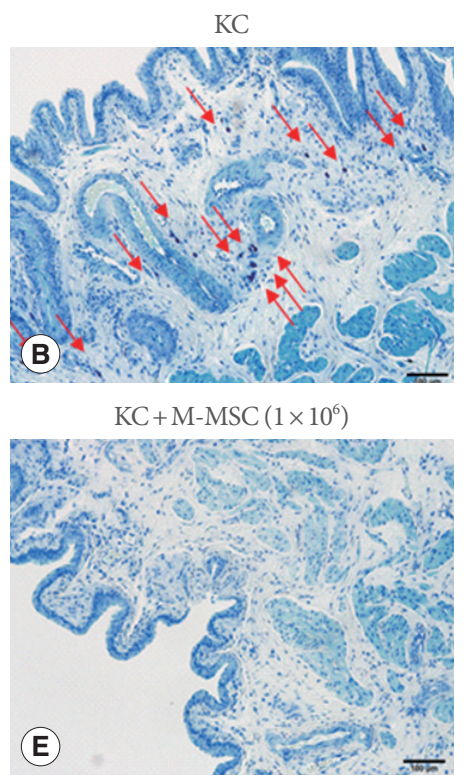
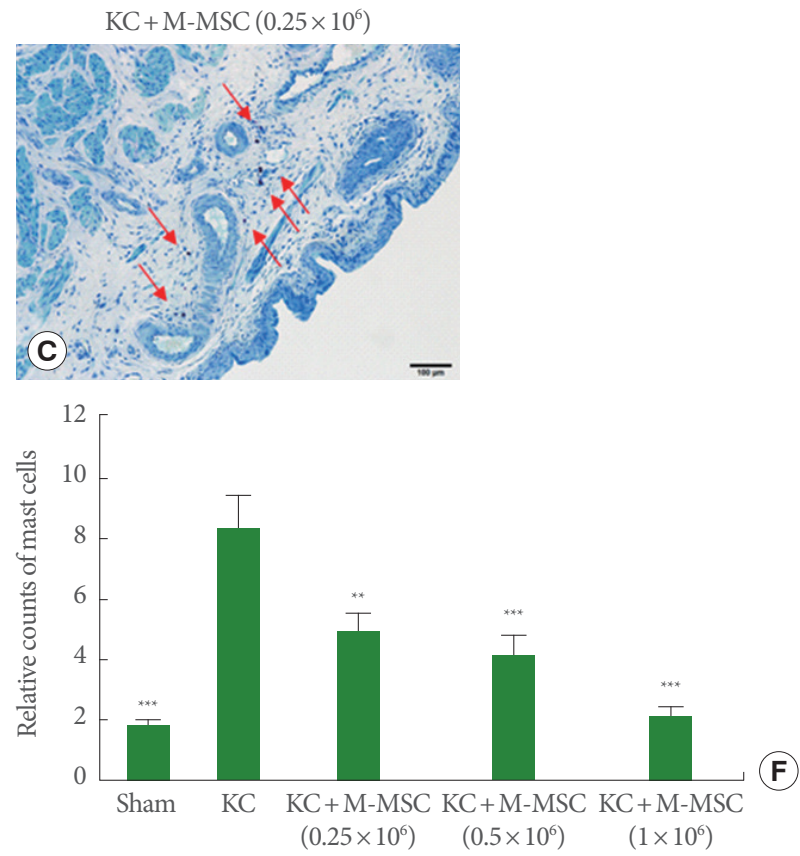

Fig. 4. The staining for mast cells. (A-E) The infiltrated mast cells in bladder tissues were stained with Toluidine Blue (red arrows) (magnification, $\times 100$; scale bar $=100 \mu \mathrm{m}$ ). (F) Relative counts of mast cells were displayed in a bar graph. KC, ketamine-induced cystitis; M-MSC, multipotent mesenchymal stem cell. ${ }^{* *} \mathrm{P}<0.01,{ }^{* * *} \mathrm{P}<0.001$ compared with the KC group with Bonferroni posttest.

and more TUNEL-stained apoptotic cells (Fig. 5) compared to the sham group. Administration of M-MSCs significantly reversed mast cell infiltration and apoptosis in KC bladders (Figs. 4, 5). Furthermore, M-MSCs prevented bladder tissue fibrosis
(Fig. 6), which is generally considered an important feature of KC. On gross histological examination, urothelial denudation and inflammation were hardly found in the bladders of either group (Fig. 7). Together, the results show that a single injection 

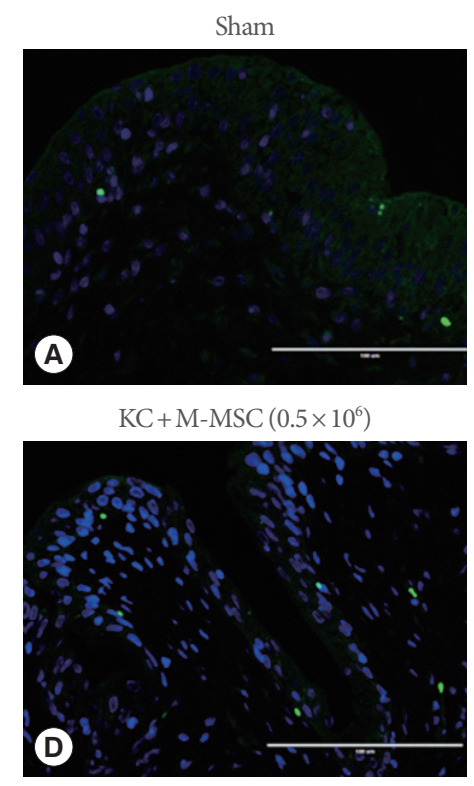
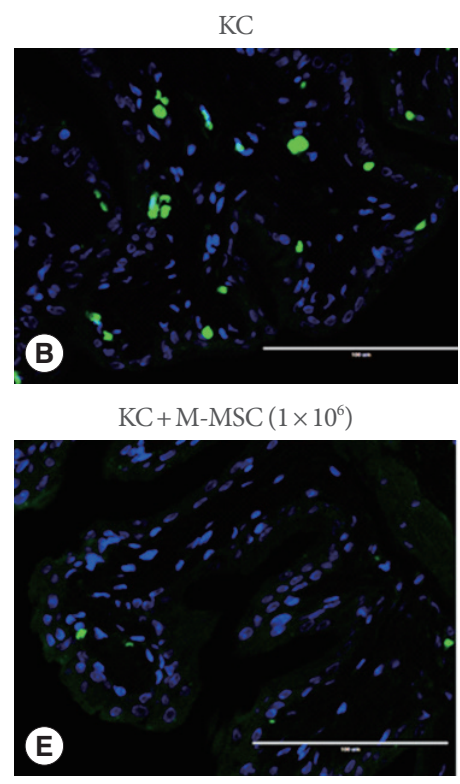
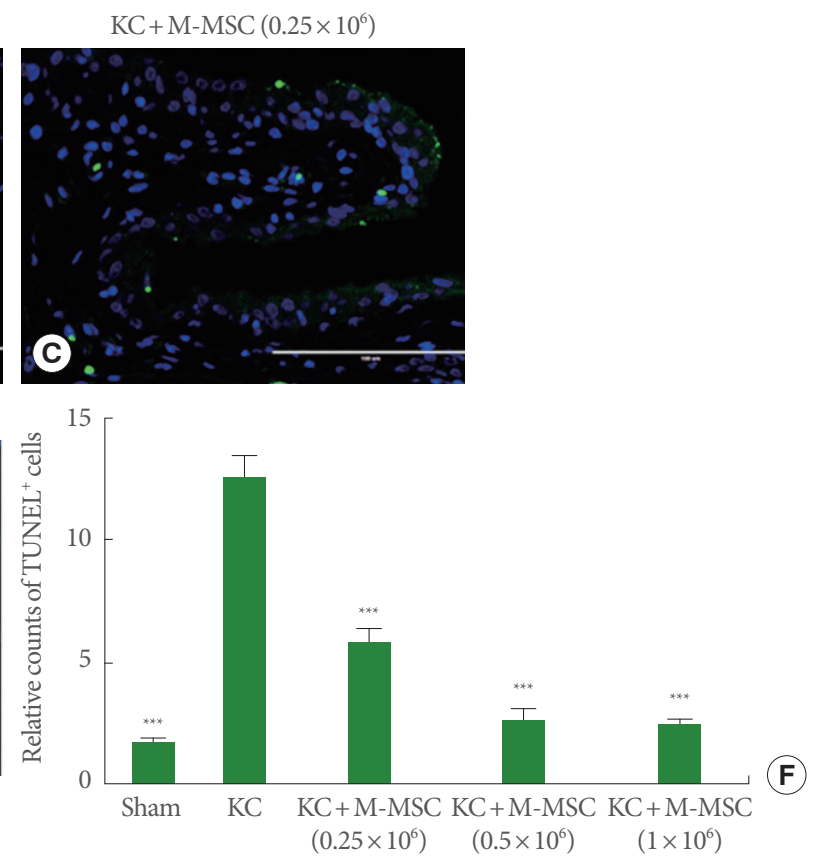

Fig. 5. TUNEL staining. (A-E) The apoptotic cells (green) in the bladder sections were stained with a TUNEL assay (magnification, $\times 400$; scale bar $=100 \mu \mathrm{m}$ ). (F) Relative counts of TUNEL cells were graphed. TUNEL, terminal dUTP nick-end labeling; KC, ketamineinduced cystitis; M-MSC, multipotent mesenchymal stem cell. ${ }^{* * *} \mathrm{P}<0.001$ compared with the KC group with Bonferroni posttest.
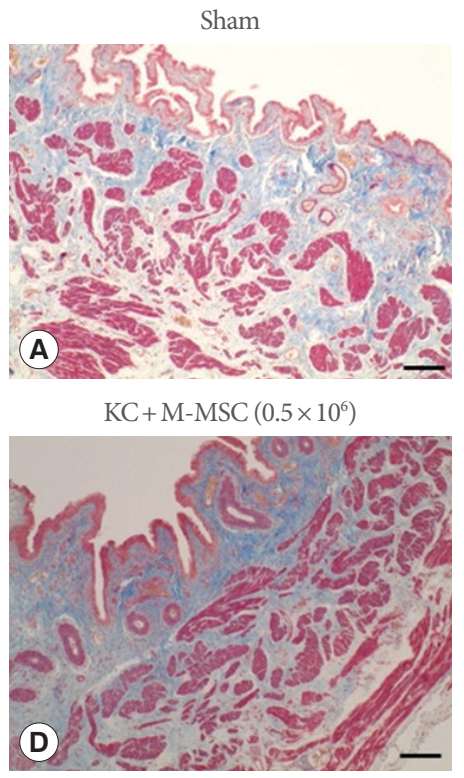
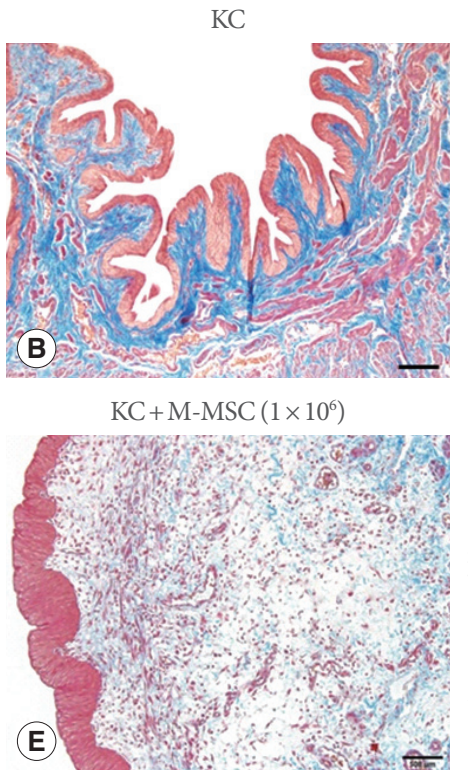
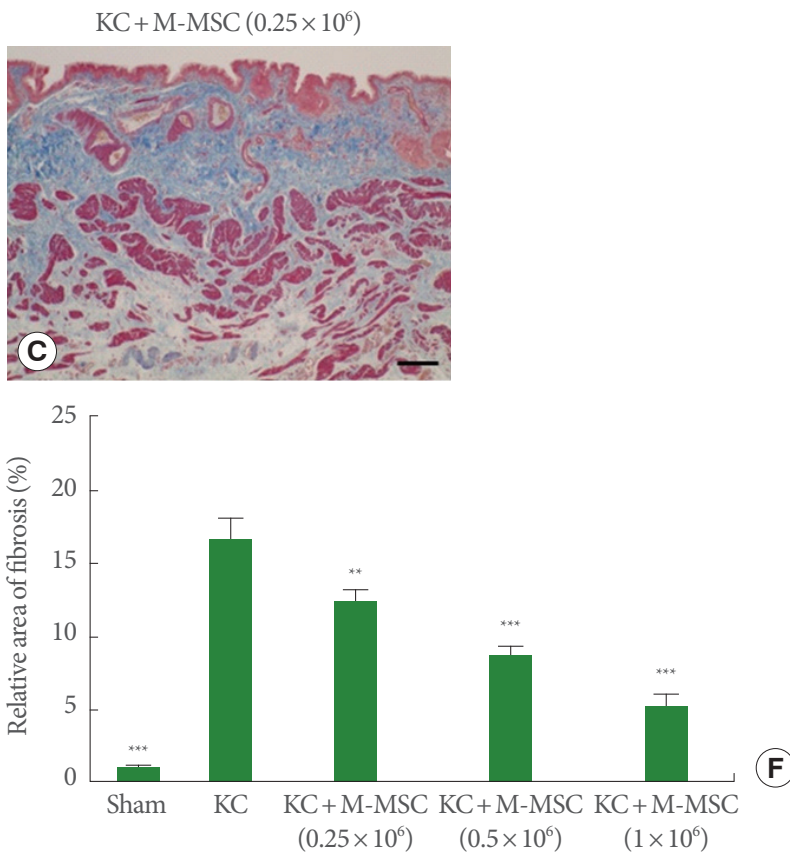

Fig. 6. The staining for detection of the fibrotic changes. (A-E) Fibrosis in the bladder sections was stained with Masson's Trichrome stain (magnification, $\times 100$; scale bar $=100 \mu \mathrm{m}$ ). The blue color indicates fibrosis. $(\mathrm{F})$ Fibrosis was quantified by digital image analysis. KC, ketamine-induced cystitis; M-MSC, multipotent mesenchymal stem cell. ${ }^{* *} \mathrm{P}<0.01,{ }^{* * *} \mathrm{P}<0.001$ compared with the KC group with Bonferroni posttest. 


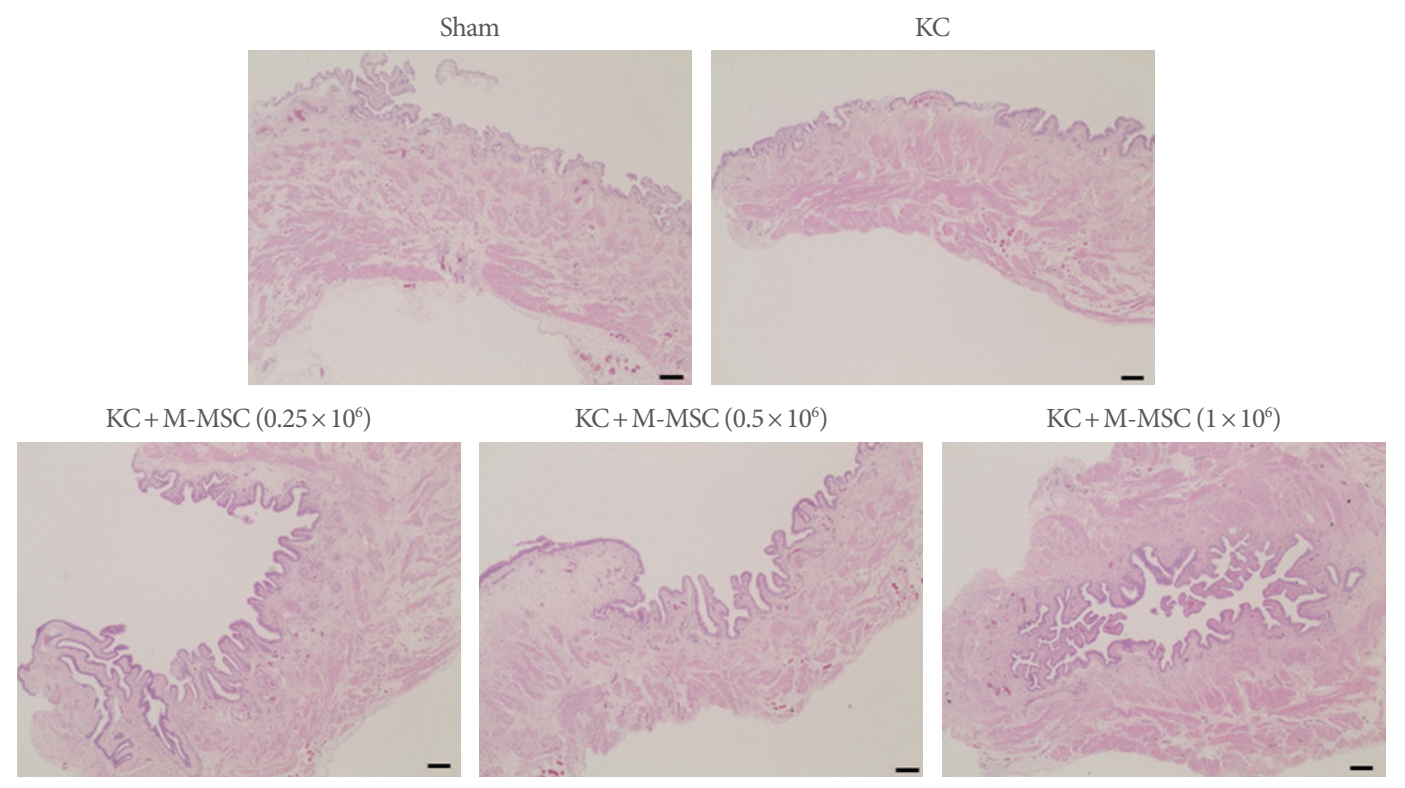

Fig. 7. Histological examination for assessing the urothelial integrity and inflammation. Hematoxylin and Eosin staining was used to evaluate the integrity of urothelium and the inflammation (magnification, $\times 40$; scale bar $=100 \mu \mathrm{m}$ ). There was no difference between the groups with regard to the integrity of urothelium and the inflammation. Significant denudation of urothelium and inflammation were scarcely found in the bladders of either group. KC, ketamine-induced cystitis; M-MSC, multipotent mesenchymal stem cell.

of M-MSCs into the bladder helps to treat KC bladder by protecting against the abnormal changes in mast cell infiltration, apoptosis and fibrotic damage, in a dose dependent manner.

\section{Gene Expression Analysis by Real-Time Quantitative-PCR}

Gene expression analysis indicated that several genes associated with tissue fibrosis, including those encoding transforming growth factor beta-1, and -2 (TGF- $\beta 1$ and - $\beta 2$ ), SMAD family members 2 and 3 (Smad2 and Smad3), and snail family zinc finger 2 (Snai2), were markedly upregulated in KC bladders. However, their expression levels were significantly suppressed after injection of M-MSCs (Fig. 8A), which indicates that such cells help to repress fibrotic damage seen in KC bladders.

Of genes associated with inflammation, that encoding tumor necrosis factor alpha was upregulated in KC bladders. However, we found no significant between-group difference in terms of the expression levels of the chemokine C-X-C motif ligand 10 (Cxcl10) or interleukin (IL)-4, although KC bladders had higher levels of the relevant transcripts. On the other hand, the expression level of IL-10, an anti-inflammatory cytokine, was markedly upregulated in bladders of the $\mathrm{KC}+\mathrm{M}-\mathrm{MSC}$ group (Fig. 8B), which suggests that a single injection of M-MSCs mitigated the inflammation induced by KC injury.

Caspase recruitment domain family member 10 (Card10), which plays a role in apoptosis, was not significantly upregulated in the KC group.

\section{DISCUSSION}

The animal studies using KC models have great significance in that they might also provide clues as to the pathophysiology of IC and help develop the novel therapeutic options for both $\mathrm{KC}$ and IC. Recently, Song et al. [30] described induction dose-dependent cystometric and fibrotic changes associated with $\mathrm{KC}$. Using a rat $\mathrm{KC}$ model, the same research team was the first to report that MSCs helped to cure KC by protecting against tissue fibrosis [16]. Daily ketamine injection over 2 weeks induced KC in Sprague-Dawley rats. KC bladders were characterized by severe mast cell infiltration, tissue fibrosis, apoptosis, and reduced bladder function. A single administration of UCB-derived MSCs into bladder tissue not only significantly ameliorated the cystometric parameters, but also reversed the characteristic histological and gene expression changes [16].

As $\mathrm{KC}$ is associated principally with long-time ketamine abuse, a limitation of the previous study [16] is the short period ( 2 weeks) over which ketamine was injected. The bladders may have been affected in a manner somewhat distinct from the bladders of actual KC patients. Thus, we developed a long-term 

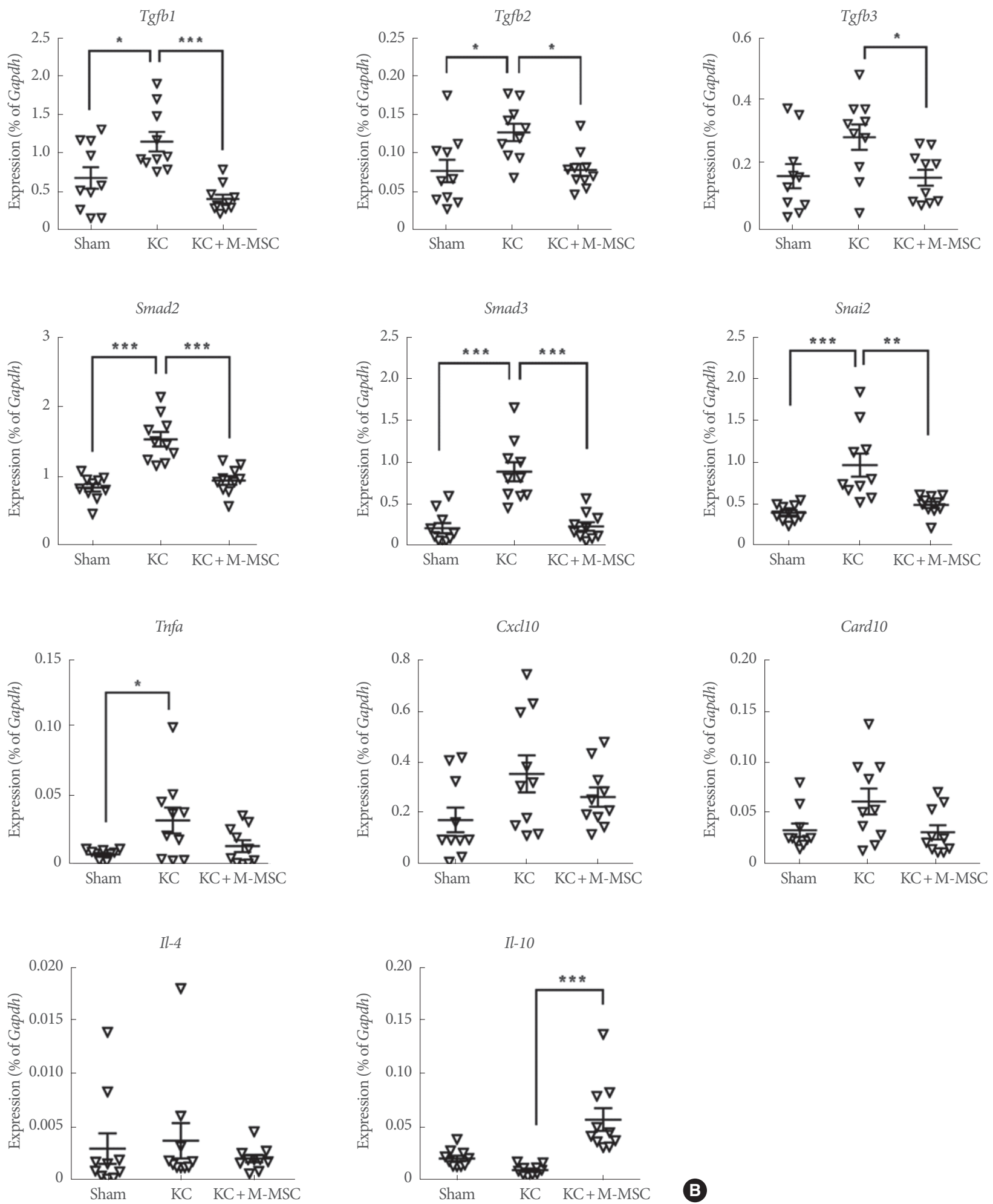

Fig. 8. Real-time quantitative polymerase chain reaction analysis of fibrosis (A) and inflammation (B)-related genes in the indicated bladder tissues. Expression is presented as \% Gapdh and shown as dot plot with the mean \pm standard error of the mean $\left({ }^{*} \mathrm{P}<0.05\right.$, ${ }^{* *} \mathrm{P}<0.01$, and ${ }^{* * *} \mathrm{P}<0.001$ compared with KC group). KC, ketamine-induced cystitis; M-MSC, multipotent mesenchymal stem cell. 
KC model; ketamine was given over 12 weeks.

The bladders of the long-term $\mathrm{KC}$ model did not differ greatly from those of the short-term model. Rats injected with ketamine over 12 weeks exhibited severely decreased storage function of the bladder. They were also featured by mast cell infiltration, fibrosis, and apoptosis. We found that transplantation of M-MSCs reversed all abnormal cystometric parameters and prevented mast cell infiltration, apoptosis, and fibrosis of $\mathrm{KC}$ bladders in a dose-dependent manner.

The pathogenesis of $\mathrm{KC}$ is complicated, involving many different pathways. Jhang et al. [5] suggested that the pathological changes in KC might be caused by persistent bladder inflammation, followed by collagen accumulation and fibrosis. However, in the present study, denudation of the urothelium and inflammation were barely evident in KC bladders, but fibrotic changes were detected. Such results again emphasize that the pathogenesis of $\mathrm{KC}$ is complicated and the pathological findings vary greatly. Indeed, several previous studies have reported contradictory results in terms of induction of urothelial degeneration in the bladders of ketamine-treated rats [31,32]. These contradictory results might be due to the different protocols used to induce KC injury. Rajandram et al. [33] considered that disruption of urothelial barrier function might not be the major pathogenic mechanism in $\mathrm{KC}$.

Large-scale production of MSCs is desirable for the development of cellular therapies for various medical conditions. MSCs derived from hESCs (M-MSCs) may serve as an unlimited source for MSCs [23,24]. Moreover, M-MSCs exhibit prolonged proliferative capacities, overcoming the lack of availability of MSCs from adult tissues. A recent study provided the first evidence of therapeutic efficacy of hESC progeny for treating urologic disorders [34]. They showed efficacy of M-MSCs in IC animal model established by hydrochloric acid instillation.

In this study, we demonstrated the therapeutic efficacy of hESC-derived M-MSCs on KC in rats. Importantly, at a low dose, M-MSC had greater beneficial effect on bladder function than did BM-MSCs. Superior potency of M-MSCs might be attributable to enhanced in vivo engraftment and survival [34], both of which are critical challenges for therapies based on MSCs from adult tissues. In several preclinical and clinical trials using adult-tissue derived MSCs, poor engraftment and survival of the transplanted cells under in vivo conditions have provoked skepticism with regard to MSC therapy. The improved engraftment of M-MSCs observed in IC animal models led to their functional integration into bladders to regenerate the damaged urothelium and also to suppress the provoked inflammatory insults [34]. A further study is required to investigate the mechanistic insight about the enhanced therapeutic capacity of M-MSCs in treating KC.

Potentially serious safety issues associated with hESC-based therapy must be addressed, including the possible formation of teratoma and other tumors, potential immune reactions, and the risk that the cell will differentiate into unwanted cell types. Adequate assessment of the biological and molecular properties of engrafted cells in pathological environments is essential for the development of effective and safe stem cell-based therapies. Therefore, further studies are needed to assess long-term safety, graft survival, and the in vivo properties of M-MSCs used to treat KC or IC. Recently, Kim et al. [34] monitored long-term safety of transplanted M-MSCs in living animals.

Another limitation of our study is that we did not assess bladder pain. BPS/IC and KC are both chronic painful bladder conditions. IC was originally considered a bladder disease, but $\mathrm{BPS} / \mathrm{IC}$ is now considered a chronic pain syndrome [35]. In patients with BPS/IC, chronic neuropathic pain may continue after the resolution of tissue damage, reflecting the fact that a maladaptive mechanism remains in play. This may also be true of patients with $\mathrm{KC}$. Central sensitization may be involved in the pathogenesis of both $\mathrm{KC}$ and BPS/IC. Pain is a subjective sensation; surrogate and behavioral markers of pain can be measured in animal models [36]. Visceral pain, such as pain emanating from the bladder, may manifest as cutaneous hypersensitivity that is quantifiable by mechanical allodynia [37]. In animal models of $\mathrm{KC}$ or IC, assessment of pain and evaluation of the peripheral and central mechanism in play could help further elucidate the pathophysiology of these conditions and the efficacy of stem cell-based therapies.

In conclusion, our findings demonstrate for the first time the therapeutic efficacy of M-MSCs on KC in rats. The injection of M-MSCs restored bladder function more effectively than did BM-derived MSCs, protecting against abnormal changes in mast cell infiltration, apoptosis and fibrotic damage. Though further studies are necessary, M-MSCs might be a promising option to treat KC.

\section{AUTHOR CONTRIBUTION STATEMENT}

- Full access to all the data in the study and takes responsibility for the integrity of the data and the accuracy of the data analysis: Sang Wook Lee, Chae-Min Ryu, Dong-Myung Shin, Myung 
-Soo Choo

- Study concept and design: Dong-Myung Shin, Myung-Soo Choo - Acquisition of data: Sang Wook Lee, Chae-Min Ryu, Jung-Hyun Shin, Daeheon Choi, Aram Kim, Hwan Yeul Yu, Ju-Young Han, Hye-Yeon Lee, Jisun Lim, Yong Hwan Kim, Jinbeom Heo, Seungun Lee, Hyein Ju, Sujin Kim, Ji-Yeon Han, Miho Song, Ki-Sung Hong, Hyung-Min Chung, Jun Ki Kim

- Analysis and interpretation of data: Sang Wook Lee, Chae-Min Ryu, Aram Kim, Hwan Yeul Yu, Dong-Myung Shin, Myung-Soo Choo

- Drafting of the manuscript: Sang Wook Lee, Chae-Min Ryu

- Critical revision of the manuscript for important intellectual content: Dong-Myung Shin, Myung-Soo Choo

- Statistical analysis: Sang Wook Lee, Chae-Min Ryu, Hwan Yeul $\mathrm{Yu}$

- Obtained funding: Dong-Myung Shin, Myung-Soo Choo

- Administrative, technical, or material support: Ki-Sung Hong, Hyung-Min Chung, Jun Ki Kim, Dong-Myung Shin, Myung-Soo Choo

- Study supervision: Dong-Myung Shin, Myung-Soo Choo

\section{REFERENCES}

1. Bokor G, Anderson PD. Ketamine: an update on its abuse. J Pharm Pract 2014;27:582-6.

2. Winstock AR, Mitcheson L, Gillatt DA, Cottrell AM. The prevalence and natural history of urinary symptoms among recreational ketamine users. BJU Int 2012;110:1762-6.

3. Jhang JF, Birder LA, Chancellor MB, Kuo HC. Patient characteristics for different therapeutic strategies in the management ketamine cystitis. Neurourol Urodyn 2017;36:687-91.

4. Chen IC, Lee MH, Chen WC, Hu TC, Lin HY. Risk factors of lower urinary tract syndrome among ketamine users. Low Urin Tract Symptoms 2017 Jul 13 [Epub]. https://doi.org/10.1111/luts.12178.

5. Jhang JF, Hsu YH, Kuo HC. Possible pathophysiology of ketaminerelated cystitis and associated treatment strategies. Int J Urol 2015; 22:816-25

6. Chu PS, Ma WK, Wong SC, Chu RW, Cheng CH, Wong S, et al. The destruction of the lower urinary tract by ketamine abuse: a new syndrome? BJU Int 2008;102:1616-22.

7. Lai Y, Wu S, Ni L, Chen Z, Li X, Yang S, et al. Ketamine-associated urinary tract dysfunction: an underrecognized clinical entity. Urol Int 2012;89:93-6.

8. Jiang S, Xie K, Cai YB. Treatment ketamine-related bladder dysfunction by intravesical injection of botulinum toxin A. J Third Mil
Med Univ 2012;34:1120-2.

9. Chung SD, Wang CC, Kuo HC. Augmentation enterocystoplasty is effective in relieving refractory ketamine-related bladder pain. Neurourol Urodyn 2014;33:1207-11.

10. Damien P, Allan DS. Regenerative therapy and immune modulation using umbilical cord blood-derived cells. Biol Blood Marrow Transplant 2015;21:1545-54.

11. Lim JY, Im KI, Lee ES, Kim N, Nam YS, Jeon YW, et al. Enhanced immunoregulation of mesenchymal stem cells by IL-10-producing type 1 regulatory T cells in collagen-induced arthritis. Sci Rep 2016; 6:26851.

12. Zhao W, Zhang C, Jin C, Zhang Z, Kong D, Xu W, et al. Periurethral injection of autologous adipose-derived stem cells with controlled-release nerve growth factor for the treatment of stress urinary incontinence in a rat model. Eur Urol 2011;59:155-63.

13. Wezel F, Southgate J, Thomas DF. Regenerative medicine in urology. BJU Int 2011;108:1046-65.

14. Gnecchi M, Zhang Z, Ni A, Dzau VJ. Paracrine mechanisms in adult stem cell signaling and therapy. Circ Res 2008;103:1204-19.

15. Song M, Lim J, Yu HY, Park J, Chun JY, Jeong J, et al. Mesenchymal stem cell therapy alleviates interstitial cystitis by activating wnt signaling pathway. Stem Cells Dev 2015;24:1648-57.

16. Kim A, Yu HY, Heo J, Song M, Shin JH, Lim J, et al. Mesenchymal stem cells protect against the tissue fibrosis of ketamine-induced cystitis in rat bladder. Sci Rep 2016;6:30881.

17. Kim A, Hoe KO, Shin JH, Choo MS. Evaluation of the incidence and risk factors associated with persistent frequency in interstitial cystitis/bladder pain syndrome and the efficacy of antimuscarinic treatment. Investig Clin Urol 2017;58:353-8.

18. Jaing TH. Umbilical cord blood: a trustworthy source of multipotent stem cells for regenerative medicine. Cell Transplant 2014;23:493-6.

19. Knaän-Shanzer S. Concise review: the immune status of mesenchymal stem cells and its relevance for therapeutic application. Stem Cells 2014;32:603-8.

20. Karlsson C, Emanuelsson K, Wessberg F, Kajic K, Axell MZ, Eriksson PS, et al. Human embryonic stem cell-derived mesenchymal progenitors: potential in regenerative medicine. Stem Cell Res 2009;3:39-50.

21. Thomson JA, Itskovitz-Eldor J, Shapiro SS, Waknitz MA, Swiergiel JJ, Marshall VS, et al. Embryonic stem cell lines derived from human blastocysts. Science 1998;282:1145-7.

22. Moon SH, Kim JM, Hong KS, Shin JM, Kim J, Chung HM. Differentiation of hESCs into mesodermal subtypes: vascular-, hematopoietic- and mesenchymal-lineage cells. Int J Stem Cells 2011;4:2434.

23. Kim JM, Hong KS, Song WK, Bae D, Hwang IK, Kim JS, et al. Peri- 
vascular progenitor cells derived from human embryonic stem cells exhibit functional characteristics of pericytes and improve the retinal vasculature in a rodent model of diabetic retinopathy. Stem Cells Transl Med 2016;5:1268-76.

24. Hong KS, Bae D, Choi Y, Kang SW, Moon SH, Lee HT, et al. A porous membrane-mediated isolation of mesenchymal stem cells from human embryonic stem cells. Tissue Eng Part C Methods 2015;21:322-9.

25. Lee T, Yoon SM. The role of intra-abdominal pressure measurement in awake rat cystometry. Int Neurourol J 2013;17:44-7.

26. Jin LH, Shin HY, Kwon YH, Park CS, Yoon SM, Lee T. Urodynamic findings in an awake chemical cystitis rat model observed by simultaneous registrations of intravesical and intraabdominal pressures. Int Neurourol J 2010;14:54-60.

27. Jin HJ, Lee HJ, Heo J, Lim J, Kim M, Kim MK, et al. Senescence-associated MCP-1 secretion is dependent on a decline in BMI1 in human mesenchymal stromal cells. Antioxid Redox Signal 2016;24:471-85.

28. Heo J, Lim J, Lee S, Jeong J, Kang H, Kim Y, et al. Sirt1 regulates DNA methylation and differentiation potential of embryonic stem cells by antagonizing Dnmt3l. Cell Rep 2017;18:1930-45.

29. Jeong EM, Yoon JH, Lim J, Shin JW, Cho AY, Heo J, et al. Real-time monitoring of glutathione in living cells reveals that high glutathione levels are required to maintain stem cell function. Stem Cell Reports 2018 Jan 3 [Epub]. pii: S2213-6711(17)30555-6. https:// doi.org/10.1016/j.stemcr.2017.12.007.

30. Song M, Yu HY, Chun JY, Shin DM, Song SH, Choo MS, et al. The fibrosis of ketamine, a noncompetitive N-methyl-d-aspartic acid receptor antagonist dose-dependent change in a ketamine-induced cystitis rat model. Drug Chem Toxicol 2016;39:206-12.

31. Shen CH, Wang ST, Lee YR, Liu SY, Li YZ, Wu JD, et al. Biological effect of ketamine in urothelial cell lines and global gene expression analysis in the bladders of ketamine injected mice. Mol Med Rep 2015;11:887-95.

32. Gu D, Huang J, Shan Z, Yin Y, Zheng S, Wu P. Effects of long-term ketamine administration on rat bladder protein levels: a proteomic investigation using two-dimensional difference gel electrophoresis system. Int J Urol 2013;20:1024-31.

33. Rajandram R, Ong TA, Razack AH, MacIver B, Zeidel M, Yu W. Intact urothelial barrier function in a mouse model of ketamineinduced voiding dysfunction. Am J Physiol Renal Physiol 2016;310: F885-94.

34. Kim A, Yu HY, Lim J, Ryu CM, Kim YH, Heo J, et al. Improved efficacy and in vivo cellular properties of human embryonic stem cell derivative in a preclinical model of bladder pain syndrome. Sci Rep 2017;7:8872.

35. Janicki TI. Chronic pelvic pain as a form of complex regional pain syndrome. Clin Obstet Gynecol 2003;46:797-803.

36. Rosen JM, Klumpp DJ. Mechanisms of pain from urinary tract infection. Int J Urol 2014;21 Suppl 1:26-32.

37. Barrot M. Tests and models of nociception and pain in rodents. Neuroscience 2012;211:39-50. 